\title{
Caractéristiques socio-démographiques et médicales influençant les qualités attribuées au bon médecin : Perspectives pour la formation à la relation médecin-patient
}

Socio-demographic and medical characteristics having an impact on the qualities attributed to competent physicians: Outlook for physician-patient relationship training

Gregory REIX ${ }^{1}$, Julien LE BRETON ${ }^{2}$, Julie CHASTANG ${ }^{3}$, Erik BERNARD ${ }^{4}$, Eric PAUTAS ${ }^{5}$, Claire RONDET ${ }^{3}$, Anne-Marie MAGNIER ${ }^{3,6}$, Gladys IBANEZ ${ }^{3,6}$

Manuscrit reçu le 15 septembre 2014 ; commentaires éditoriaux formulés aux auteurs le 13 février et le $1^{\mathrm{er}}$ mai 2015 ; accepté pour publication le 2 mai 2015

1 Département de Médecine Générale, Faculté de Médecine, Université Bordeaux Segalen, 146 rue Léo-Saignat, 33076 Bordeaux Cedex, France

2 Université Paris Est (UPEC), Faculté de Médecine, Département de Médecine Générale, Faculté de Médecine, LIC E4393, 94000 Créteil, France

3 Département de Médecine Générale, Faculté de Médecine, Université Pierre et Marie Curie (UPMC), Paris Sorbonnes, 27 rue de Chaligny, 75012 Paris, France

4 Département de Médecine Générale PIFO, Université Versailles Saint-Quentin en Yvelines, 78280 Guyancourt, France Email : bernard.erik@gmail.com

5 Unité Gériatrique Aiguë Hôpital Charles Foix, GH Pitié-Salpêtrière-Charles Foix (APHP), 7 avenue de la République, 94205 Ivry-sur-Seine, France

6 SFTG, Société de Formation Thérapeutique du Généraliste, 233 bis rue de Tolbiac, 75013 Paris, France

Mots-clés
Étude transversale ;
Satisfaction
du patient ;
Relations médecin-
malade ; Compétence
professionnelle ;
Observance
de la prescription

Mots-clés

Etude transversale

Relations médecinde la prescription
Résumé - Contexte : La satisfaction du patient, au sein d'une relation médecin-malade de qualité, améliore son observance, son adhésion thérapeutique et in fine son état de santé. L'objectif de cette étude était d'analyser les facteurs influençant les qualités attribuées par les patients au bon médecin. Méthode : Enquête transversale sous la forme d'un questionnaire téléphonique, élaboré après une étude pilote, auprès de patients majeurs. Douze qualités attribuées au bon médecin dans les domaines médicaux, relationnels, éthiques ou organisationnels ont été étudiées. Des facteurs d'ordre sociodémographiques, liés au parcours médical ou à l'état de santé des patients ont été pris en compte. Une déclaration à la CNIL a été déposée. Les analyses descriptives, univariées et multivariées ont été réalisées à l'aide du logiciel SAS 9.1. 


\begin{tabular}{|c|c|}
\hline & $\begin{array}{l}\text { Résultats : Sur } 1246 \text { appels téléphoniques, } 302 \text { patients ont été inclus. La capacité à réaliser } \\
\text { un bon diagnostic, l'écoute et l'intégrité du médecin étaient les qualités les plus citées. La pré- } \\
\text { sence d'une maladie chronique était associée à des exigences d'écoute et de qualités relation- } \\
\text { nelles accrues, selon un gradient de sévérité de la maladie et après ajustement multivarié. } \\
\text { D'autres facteurs sociodémographiques ou liés à l'expérience médicale du patient semblaient } \\
\text { influer sur les qualités attendues du bon médecin. Conclusion : Ces résultats soulignent } \\
\text { l'importance des qualités relationnelles dans la relation médecin malade. Celles-ci sont à pro- } \\
\text { mouvoir et à enrichir tout au long du cursus médical et de la carrière de soignant. }\end{array}$ \\
\hline $\begin{array}{l}\text { Keywords } \\
\text { Cross-sectional } \\
\text { survey; Patient } \\
\text { satisfaction; } \\
\text { Physician-patient } \\
\text { relationship; } \\
\text { Professional } \\
\text { competence; } \\
\text { Compliance } \\
\text { with prescriptions }\end{array}$ & $\begin{array}{l}\text { Abstract - Background: In the context of an efficient physician-patient relationship, } \\
\text { patient satisfaction improves observance, adherence and ultimately health. Ojective: The } \\
\text { objective of this study was to analyze the factors having an impact on the qualities attributed } \\
\text { by patients to competent physicians. Method: A cross-sectional phone survey using a ques- } \\
\text { tionnaire was developed as a result of a pilot study with major patients. Twelve qualities } \\
\text { attributed to competent physicians in the medical, relational, ethical and organizational } \\
\text { fields were examined. Sociodemographic factors related to patient medical history or health } \\
\text { status were taken into account. A declaration to the Commision nationale de l'informatique } \\
\text { et des libertés (National commssion on computer science and freedoms) was filed. Descrip- } \\
\text { tive, univariate and multivariate analyzes were performed using SAS } 9.1 \text { software. Results: } \\
302 \text { patients were included following the } 1,246 \text { phone calls. The physician's ability to per- } \\
\text { form an accurate diagnosis, actively listen and his/her integrity were the qualities mentioned } \\
\text { most often. The incidence of a chronic disease was associated with better listening and } \\
\text { interpersonal skills, based on a disease severity gradient and following multivariate adjust- } \\
\text { ment. Other sociodemographic factors and some related to the patients' medical experience } \\
\text { seemed to have an impact on the qualities expected of competent physicians. Conclusion: } \\
\text { These findings underscore the importance of interpersonal skills in the physician-patient } \\
\text { relationship. These need to be promoted and broadened throughout the medical curriculum } \\
\text { and throughout the caregiving career. }\end{array}$ \\
\hline
\end{tabular}

\section{Introduction}

En 1995, l'Organisation Mondiale de la Santé a défini la responsabilité sociale en éducation médicale comme l'obligation pour les facultés de médecine de répondre aux besoins prioritaires en matière de santé des collectivités qu'elles desservent ${ }^{[1]}$. Au cours des dernières décennies, la formation médicale a insisté sur la compétence technique et la spécialisation. Au regard d'une telle perspective, les médecins étaient surtout formés dans une approche centrée sur le médecin plutôt qu'une approche centrée sur la communauté et le patient ${ }^{[2]}$. Répondre aux besoins et aux attentes de la communauté nécessite de se préoccuper de la satisfaction des patients, dans une relation de soin de qualité.
La relation médecin-patient est une rencontre entre deux individus, le plus souvent dans un colloque «singulier»; c'est une relation d'aide et d'échange. Lorsqu'elle est de qualité, cette relation augmente le niveau de satisfaction du patient, favorise son observance et son adhésion thérapeutique ${ }^{[3]}$. Par ailleurs, la croyance du patient d'avoir en face de lui un bon médecin accroît l'effet placebo du médecin et ainsi l'efficacité du traitement prescrit ${ }^{[4]}$. Cette relation de qualité présente aussi des intérêts pour le médecin en prévenant les conflits avec les patients par une meilleure adéquation des attentes respectives. En outre, elle serait un facteur protecteur du syndrome d'épuisement professionnel ${ }^{[5]}$.

Cerner les attentes du patient suppose une connaissance préalable de sa perception du bon 
médecin $^{[6]}$. Qu'est-ce qu'un bon médecin? Les réponses seront différentes selon les points de vue envisagés : les médecins, les patients, les autorités de santé, les instances politiques, les philosophes, les caisses d'assurance maladie peuvent avoir des conceptions qui leur sont propres ${ }^{[7-11]}$. Plusieurs travaux ont décrit les qualités attendues d'un bon médecin dans des populations variées. Selon une récente revue de la littérature, plusieurs dimensions seraient nécessaires à la définition des qualités du bon médecin : techniques, relationnelles et éthiques ${ }^{[12-14]}$. Cette réflexion pourrait être approfondie en étudiant si les facteurs individuels propres aux patients modifient ces attentes.

Cette étude avait pour objectif d'identifier les facteurs influençant les qualités attribuées par les patients au bon médecin. Les aspects sociodémographiques, le parcours médical du patient et son état de santé ont été pris en compte dans ce travail exploratoire.

\section{Méthodes}

\section{Type d'enquête et population}

Une enquête transversale a été réalisée à l'aide d'un questionnaire standardisé administré par téléphone sur une durée de deux mois. Tous les patients ayant appelé au cours de l'année la régulation des médecins libéraux de Toulouse ou la régulation de SOS médecins Toulouse ont été considérés comme éligibles pour participer à l'étude. SOS médecins est le premier réseau d'urgence et de permanence de soins en France. Il est constitué d'un milliers de médecins urgentistes en France, disponibles 24 heures sur 24, qui assurent 2,5 millions d'interventions (visites à domicile le plus souvent, ou des consultations) ${ }^{[15]}$. Les patients inclus devaient avoir plus de 18 ans, avoir déclaré un médecin traitant et avoir consulté SOS médecins au maximum une fois dans l'année pour eux-mêmes ou leur famille proche. Ces critères avaient pour but de sélectionner des patients dont le principal recours médical était leur médecin traitant. Les numéros de téléphone du domicile ou du portable ont été utilisés. Les numéros correspondant à des institutions (maison de retraite, entreprise...) n'ont pas été appelés. De même, si le motif de la visite était un certificat de décès, le numéro n'a pas été appelé. Lorsque l'appel aboutissait à une messagerie, aucun message n'était laissé et lorsque le patient était momentanément non disponible pour répondre, un rendez-vous téléphonique ultérieur était proposé. L'étude a été poursuivie jusqu'à atteindre un nombre minimal de 300 réponses au questionnaire.

\section{Questionnaire}

\section{Elaboration du questionnaire}

Un questionnaire a été construit spécifiquement pour l'étude en trois étapes. Premièrement, une revue de la littérature a été réalisée en interrogeant les bases de données MEDLINE et BDSP (Banque de données en Santé Publique) en utilisant les termes suivants : «Good doctor» or «Good physician» (aucun terme Mesh n'ayant été identifié). Puis, ce questionnaire a été soumis à l'avis de trois médecins généralistes pour adapter la formulation de certaines questions. La version intermédiaire du questionnaire a été testée auprès de 30 patients dans le cadre d'une étude pilote au sein du même registre de patients. Les résultats de cette étude ont permis de clarifier et préciser certains items, pour améliorer la qualité des données recueillies, et de vérifier la faisabilité et l'acceptabilité globale du questionnaire. La version finale du questionnaire est présenté en annexe.

\section{Données socio-démographiques}

Les données suivantes ont été recueillies auprès des patients : le sexe, l'âge, la catégorie socioprofessionnelle selon la nomenclature des professions et catégories socioprofessionnelles de l'Institut National de la Statistique et des Études Economiques (INSEE) ${ }^{[16]}$, la prise en charge éventuelle par la couverture médicale universelle complémentaire (CMUc) ou l'aide médicale d'état (AME) et le nombre d'enfants. Les dispositifs de CMUc ou de AME sont entièrement financés par l'état des soins médicaux; leur identification visait à repérer les patients en situation de fragilité sociale. 


\section{Données médicales}

Les données médicales receuillies comportaient : la fréquence annuelle de consultation chez le médecin traitant, la durée de suivi avec le médecin traitant actuel, la présence d'une affection longue durée (ALD - affections chroniques sévères dont la prise en charge financière par l'assurance maladie est totale), la liste des médicaments pris quotidiennement et leur indication. Nous avons classé l'état de santé des patients en trois catégories : les patients n'ayant pas de traitement chronique (ou seulement une contraception), les patients ayant un traitement chronique hors ALD, et les patients ayant une maladie chronique en ALD. Pour les patients ayant une (ou plusieurs) maladie(s) chronique(s), plusieurs familles de pathologies ont été créées, correspondant aux affections les plus fréquemment rencontrées: hypertension artérielle, diabète, syndrome anxieux/dépressif ou insomnie, cancer, substitution thyroïdienne, ostéoporose, dyslipidémie, pathologie cardiovasculaire, allergie, pathologie psychiatrique (autre qu'anxiodépressive ou insomnie), autres maladies chroniques.

\section{Qualités du bon médecin}

Le questionnaire comportait 12 qualités attribuables au bon médecin, inspirées des différents domaines décrits dans la littérature ${ }^{[17,18]}$ : les qualités médicales (capacité diagnostique et de traitement, respect des recommandations, formation médicale continue et participation à la formation des étudiants), les qualités relationnelles (capacité d'écoute, proposition de décision partagée, conseils de prévention, coopération avec les autres soignants), les qualités éthiques (intégrité/honnêteté du médecin, indépendance vis-àvis des laboratoires pharmaceutiques) et enfin les qualités organisationnelles du médecin (accessibilité aisée à une consultation et informatisation du cabinet). L'importance de ces qualités dans la définition du bon médecin a été mesurée à l'aide d'une échelle de Likert cotée de 1 (pas ou peu important) à 4 (extrêmement important). Enfin, les questions suivantes ont été posées : rencontre passée avec un bon médecin (oui/non), rencontre passée avec un mauvais médecin (oui/non), préférence éventuelle du sexe ou de l'âge du médecin. Ces dernières questions ont eu pour objectif d'ouvrir le dialogue et de recueillir quelques opinions personnelles complémentaires.

\section{Comité d'éthique}

Une déclaration à la Commission nationale de l'informatique et des libertés (CNIL) pour autorisation d'évaluation des pratiques de soins a été déposée le 11 avril 2011. Le consentement du patient était systématiquement demandé en début d'entretien téléphonique. Les réponses ont été anonymisées à l'aide d'un identifiant numérique.

\section{Analyse statistique}

Une description de l'échantillon a été réalisée à l'aide de moyennes, écart-types et pourcentages. La consistance interne du questionnaire a été testée par le coefficient alpha de Crohnbach. Puis, les qualités attribuées au bon médecin ont été comparées selon les facteurs socio-démographiques ou médicaux des patients en utilisant les tests non-paramétriques de Mann-Whitney ou de Kruskal Wallis. Les moyennes des scores ont été décrites par groupe avec un seuil de significativité fixé à $p \leq 0,20$. L'état de santé des patients a été comparé selon les domaines de qualités citées par les patients (médical, relationnel, éthique ou organisationnel). Une modélisation multivariée a été utilisée pour illustrer l'association entre l'état de santé du patient et les qualités attribuées au bon médecin, à l'aide de modèles linéaires mixtes et après ajustement sur les principales variables sociodémographiques de l'échantillon (âge, sexe et catégorie socio-professionnelle des patients). Le seuil de significativité a été fixé à $p \leq 0,05$. Toutes les analyses statistiques ont été effectuées sur le logiciel SAS version 9.1 (Cary, NC, USA).

\section{Résultats}

\section{Description de l'échantillon}

Sur 1246 appels téléphoniques, 302 patients ont été inclus (figure 1). Le coefficient alpha de Cronbach du questionnaire était de $\alpha=0.68$. 


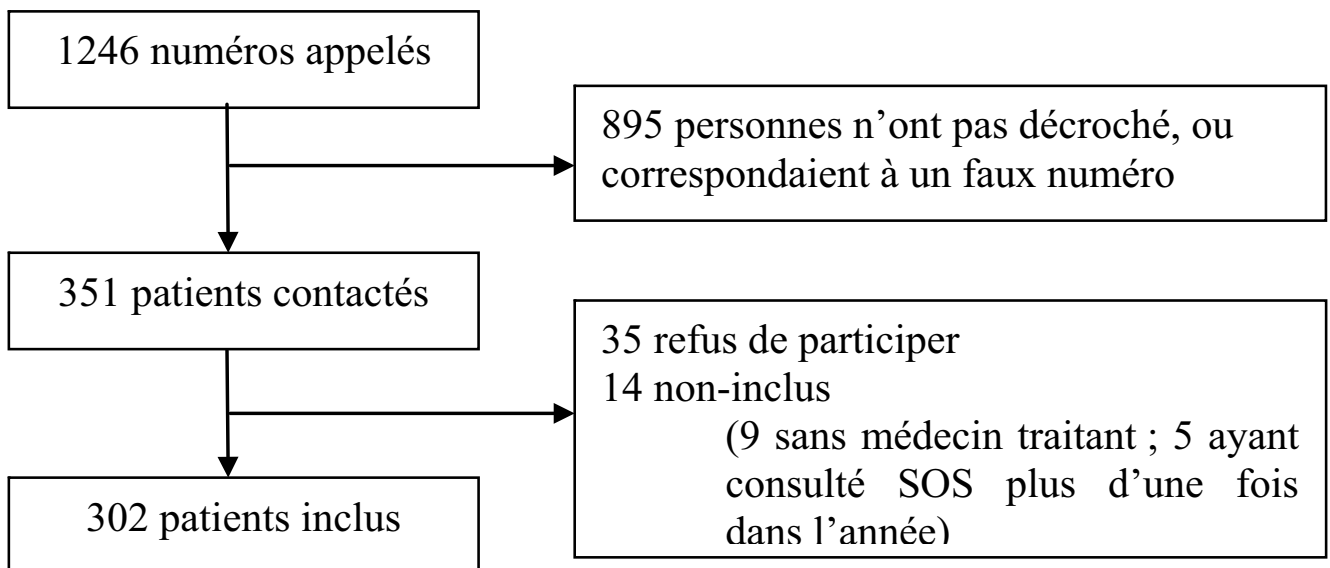

Fig. 1. Organigramme de sélection des patients dans l'étude.

L'âge moyen était de 43,5 ans $\pm 16,6$ ans et le sexe était majoritairement féminin $(70,2 \%)$ (tableau I). Dans notre échantillon, 88,4\% des patients exerçaient une profession et pour $8,6 \%$ des personnes interrogées il s'agissait d'une profession médicale ou paramédicale (médecins, dentistes, pharmaciens, sages-femmes, infirmières, masseurs kinésithérapeutes, psychologues, orthophonistes, aides-soignantes). La durée de suivi par le médecin traitant allait de 0 à 37 ans (moyenne $=8,8$ ans). $\mathrm{La}$ majorité des patients $(59,6 \%)$ ne prenaient aucun traitement régulier, $24,5 \%$ prenaient un traitement régulier sans être en ALD et 15,9\% bénéficiaient de l'ALD.

La capacité du médecin à établir un diagnostic et à traiter son malade a été la qualité la mieux notée par les patients (moyenne $=3,29 / 4$ ) (tableau II). Cette qualité se distinguait par un très fort pourcentage de réponses maximales (41\% de extrêmement important) et quasiment pas de réponses pas ou peu important $(n=2)$. Par ailleurs, sa dispersion était la plus faible de toutes les qualités (écart-type à 0,68 ). Ensuite, les qualités d'écoute du médecin (moyenne $=3,12$ ), son intégrité (moyenne $=3,03$ ) et son obligation de formation continue (moyenne $=2,97$ ) ont été les qualités les plus souvent citées, considérées en moyenne comme très importantes. La question de l'indépendance des médecins envers les laboratoires pharmaceutiques était la dernière des qualités notées (moyenne $=1,52$ ) avec près de $70 \%$ de réponse pas ou peu important.

\section{Caractéristiques socio-démographiques}

L'influence des facteurs, classés selon leur nature socio-démographique, liée à l'expérience médicale ou à l'état de santé du patient, est décrite dans les tableaux III et IV. Les patients les plus âgés accordaient plus d'importance à la coopération entre les soignants et à l'écoute de la part de leur médecin que les autres patients (tableau III). Par rang de classement, l'écoute était au premier rang des qualités notées par les patients âgés, tandis que la qualité du bon diagnostic était au premier rang des qualités citées par les personnes de 18 à 29 ans et de 30 à 59 ans.

L'accessibilité du médecin était une qualité plus importante pour les femmes que pour les hommes (moyenne $=2,62 v s .2,41 ; p=0,05$ ). Les parents souhaitaient un plus grand partage des informations entre soignants que les non-parents (moyenne $=2,56$ vs. 2,$34 ; p=0,04)$. Les parents étaient également davantage demandeurs de conseil de prévention de la part de leur médecin.

Les personnes sans profession (composées majoritairement d'étudiants dans notre échantillon, $c f$. plus haut) étaient plus sensibles à l'informatisation du cabinet que les autres personnes interrogées. De même, les personnes sans profession attribuaient 
Tableau I. Caractéristiques des patients de l'étude.

\begin{tabular}{|c|c|c|}
\hline Caractéristiques des patients & N ou moy $\pm \sigma$ & $\%$ ou intervalle \\
\hline Age & 43,5 ans $\pm 16,6$ & {$[18 ; 88]$} \\
\hline De 18 à 29 ans & 79 & 26,16 \\
\hline De 30 à 59 ans & 167 & 55,30 \\
\hline Plus de 60 ans & 56 & 18,54 \\
\hline \multicolumn{3}{|l|}{ Sexe } \\
\hline Hommes & 90 & 29,80 \\
\hline Femmes & 212 & 70,20 \\
\hline Enfants & 1,4 enfant $\pm 1,3$ & {$[0 ; 10]$} \\
\hline Patients ayant au moins un enfant & 208 & 68,87 \\
\hline \multicolumn{3}{|l|}{ Catégories socioprofessionnelles } \\
\hline Agriculteurs exploitants & 2 & 0,66 \\
\hline Artisans, commerçants et chefs d'entreprise & 9 & 2,98 \\
\hline Cadres et professions intellectuelles & 51 & 16,89 \\
\hline Professions intermédiaires & 77 & 25,50 \\
\hline Employés & 116 & 38,41 \\
\hline Ouvriers & 12 & 3,97 \\
\hline Autres personnes sans activité professionnelle & 35 & 11,59 \\
\hline Dont étudiants & 18 & 5,96 \\
\hline Exerçant une profession médicale ou paramédicale & 26 & 8,61 \\
\hline Bénéficiaires de la CMUc ou de l'AME & 34 & 11,26 \\
\hline \multicolumn{3}{|l|}{ Fréquence de consultation chez le médecin traitant } \\
\hline Une fois par an ou moins souvent & 52 & 17,39 \\
\hline Une fois tous les 6 mois environ & 74 & 24,75 \\
\hline Une fois tous les 3 mois environ & 93 & 31,00 \\
\hline Une fois tous les mois ou plus souvent & 80 & 26,67 \\
\hline Durée de suivi avec le médecin traitant actuel & 8,8 ans $\pm 8,4$ & {$[0 ; 37]$} \\
\hline \multicolumn{3}{|l|}{ État de santé } \\
\hline Patients sans maladie chronique & 180 & 59,60 \\
\hline Patients avec une maladie chronique sans ALD & 74 & 24,50 \\
\hline Patients en ALD & 48 & 15,89 \\
\hline
\end{tabular}


Tableau I. Suite.

\begin{tabular}{|c|c|c|}
\hline Caractéristiques des patients & N ou moy $\pm \sigma$ & \% ou intervalle \\
\hline \multicolumn{3}{|l|}{ Distribution des malades chroniques (avec ou sans ALD) } \\
\hline Cancer guéri ou actuel & 16 & 5,30 \\
\hline Diabète & 12 & 3,97 \\
\hline Hypertension artérielle & 39 & 12,91 \\
\hline Dyslipidémie & 14 & 4,64 \\
\hline Maladie cardiovasculaire autre & 14 & 4,64 \\
\hline Maladie psychiatrique (antipsychotique ou thymorégulateur) & 4 & 1,32 \\
\hline Dépression ou anxiété & 15 & 4,97 \\
\hline Ostéoporose & 7 & 2,33 \\
\hline Maladie thyroïdienne & 11 & 3,64 \\
\hline Allergie & 11 & 3,64 \\
\hline Autre maladie chronique & 54 & 17,88 \\
\hline
\end{tabular}

CMUc : couverture médicale universelle complémentaire; AME aide médicale d'état; ALD affection de longue durée.

Tableau II. Qualités citées d'un bon médecin. Note globale, distribution des réponses et rang de classement.

\begin{tabular}{|c|c|c|c|c|c|c|c|}
\hline \multirow[b]{2}{*}{ Qualités } & \multirow[b]{2}{*}{$N$} & \multirow{2}{*}{$\begin{array}{c}\begin{array}{c}\text { Note globale } \\
\text { obtenue }\end{array} \\
\text { Moy } \pm \sigma\end{array}$} & \multicolumn{4}{|c|}{ Répartition des réponses par qualité du bon médecin } & \multirow[t]{2}{*}{ Rang } \\
\hline & & & $\begin{array}{c}\text { Pas ou peu } \\
\text { important } \\
\%(n)\end{array}$ & $\begin{array}{c}\text { D'importance } \\
\text { moyenne } \\
\%(n)\end{array}$ & $\begin{array}{c}\text { Très } \\
\text { important } \\
\%(n)\end{array}$ & $\begin{array}{c}\text { Extrêmement } \\
\text { important } \\
\%(n)\end{array}$ & \\
\hline Diagnostic & 302 & $3,29 \pm 0,68$ & $0,66(2)$ & $10,93(33)$ & $47,35(143)$ & $41,06(124)$ & 1 \\
\hline Écoute & 302 & $3,12 \pm 0,69$ & $1,66(5)$ & $13,58(41)$ & $55,63(168)$ & $29,14(88)$ & 2 \\
\hline Intégrité & 302 & $3,03 \pm 0,76$ & $2,65(8)$ & $19,54(59)$ & $49,67(150)$ & $28,15(85)$ & 3 \\
\hline Formation continue & 300 & $2,97 \pm 0,70$ & $2,67(8)$ & $17,67(53)$ & $59,33(178)$ & $20,33(61)$ & 4 \\
\hline Accessibilité & 300 & $2,56 \pm 0,82$ & $9,00(27)$ & $38,67(116)$ & $40,00(120)$ & $12,33(37)$ & 5 \\
\hline Coopération & 298 & $2,49 \pm 0,84$ & $12,42(37)$ & $35,91(107)$ & $41,61(124)$ & $10,07(30)$ & 6 \\
\hline Conseils & 300 & $2,41 \pm 0,78$ & $12,00(36)$ & $40,67(122)$ & $41,33(124)$ & $6,00(18)$ & 7 \\
\hline Formation d'étudiants & 294 & $2,02 \pm 0,92$ & $34,35(101)$ & 35,37 (104) & $23,81(70)$ & $6,46(19)$ & 8 \\
\hline $\begin{array}{l}\text { Suivi des } \\
\text { recommandations }\end{array}$ & 295 & $1,97 \pm 0,90$ & 36,95 (109) & $33,90(100)$ & $24,07(71)$ & $5,08(15)$ & 9 \\
\hline Décision partagée & 298 & $1,94 \pm 0,83$ & $34,90(104)$ & 38,93 (116) & $23,49(70)$ & $2,68(8)$ & 10 \\
\hline Informatisation & 301 & $1,79 \pm 0,84$ & $45,51(137)$ & $32,89(99)$ & $18,94(57)$ & $2,66(8)$ & 11 \\
\hline $\begin{array}{l}\text { Indépendance } \\
\text { avec les laboratoires }\end{array}$ & 264 & $1,52 \pm 0,87$ & $68,94(182)$ & $15,15(40)$ & $11,36(30)$ & $4,55(12)$ & 12 \\
\hline
\end{tabular}

plus d'importance au suivi des recommandations que le reste de l'échantillon. Par rang de classement, la formation continue est apparue au deuxième rang d'importance et l'écoute au quatrième rang.
Les patients bénéficiaires de la CMUc ou de l'AME étaient également plus sensibles à l'informatisation du cabinet que les autres personnes interrogées, ainsi qu'au suivi des recommandations. 


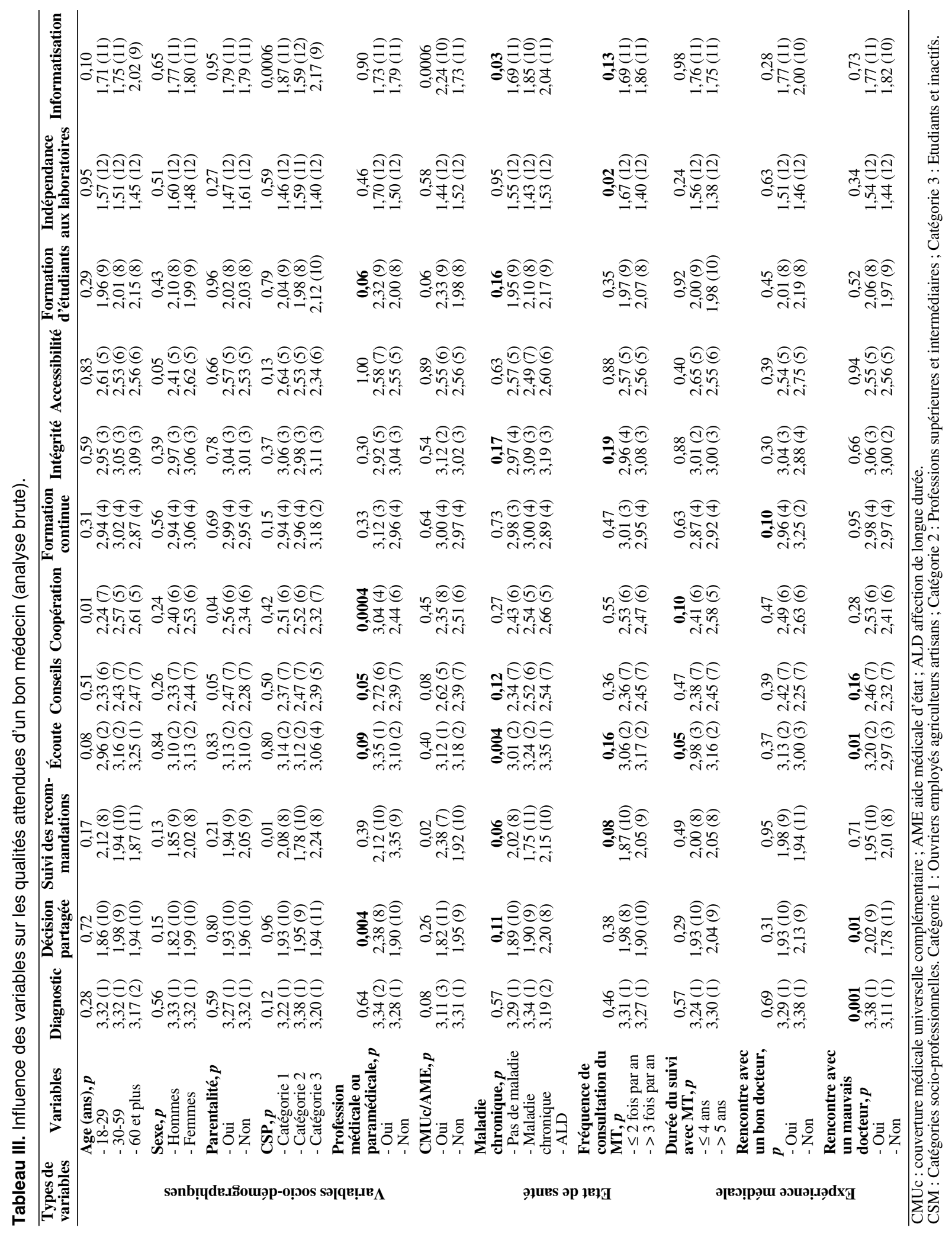


Tableau IV. Les trois principales qualités du bon médecin selon les variables étudiées.

\begin{tabular}{|c|c|c|c|c|}
\hline $\begin{array}{l}\text { Type de } \\
\text { variables }\end{array}$ & Variables & Rang 1 & Rang 2 & Rang 3 \\
\hline \multirow{6}{*}{ 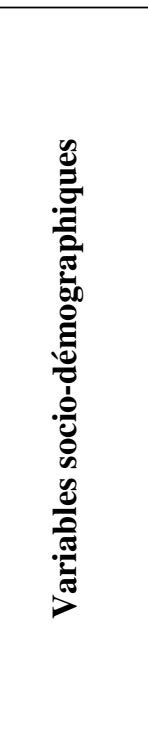 } & $\begin{array}{l}\hat{\text { Âge }} \\
-18-29 \\
-30-59 \\
-60 \text { et plus }\end{array}$ & $\begin{array}{l}\text { Diagnostic } \\
\text { Diagnostic } \\
\text { Écoute }\end{array}$ & $\begin{array}{l}\text { Écoute } \\
\text { Écoute } \\
\text { Diagnostic }\end{array}$ & $\begin{array}{l}\text { Intégrité } \\
\text { Intégrité } \\
\text { Intégrité }\end{array}$ \\
\hline & $\begin{array}{l}\text { Sexe } \\
\text { - Hommes } \\
\text { - Femmes }\end{array}$ & $\begin{array}{l}\text { Diagnostic } \\
\text { Diagnostic }\end{array}$ & $\begin{array}{l}\text { Écoute } \\
\text { Écoute }\end{array}$ & $\begin{array}{l}\text { Intégrité } \\
\text { Intégrité }\end{array}$ \\
\hline & $\begin{array}{l}\text { Parentalité } \\
\text { - Oui } \\
\text { - Non }\end{array}$ & $\begin{array}{l}\text { Diagnostic } \\
\text { Diagnostic }\end{array}$ & $\begin{array}{l}\text { Écoute } \\
\text { Écoute }\end{array}$ & $\begin{array}{l}\text { Intégrité } \\
\text { Intégrité }\end{array}$ \\
\hline & $\begin{array}{l}\text { CSP } \\
\text { - Catégorie } 1 \\
\text { - Catégorie } 2 \\
\text { - Catégorie } 3\end{array}$ & $\begin{array}{l}\text { Diagnostic } \\
\text { Diagnostic } \\
\text { Diagnostic }\end{array}$ & $\begin{array}{c}\text { Écoute } \\
\text { Écoute } \\
\text { Formation continue }\end{array}$ & $\begin{array}{l}\text { Intégrité } \\
\text { Intégrité } \\
\text { Intégrité }\end{array}$ \\
\hline & $\begin{array}{l}\text { Profession médicale ou paramédicale } \\
\text { - Oui } \\
\text { - Non }\end{array}$ & $\begin{array}{c}\text { Écoute } \\
\text { Diagnostic }\end{array}$ & $\begin{array}{l}\text { Diagnostic } \\
\text { Écoute }\end{array}$ & $\begin{array}{l}\text { Formation continue } \\
\text { Intégrité }\end{array}$ \\
\hline & $\begin{array}{l}\text { CMUc / AME } \\
\text { - Oui } \\
\text { - Non }\end{array}$ & $\begin{array}{c}\text { Écoute } \\
\text { Diagnostic }\end{array}$ & $\begin{array}{l}\text { Intégrité } \\
\text { Écoute }\end{array}$ & $\begin{array}{l}\text { Diagnostic } \\
\text { Intégrité }\end{array}$ \\
\hline \multirow{2}{*}{ 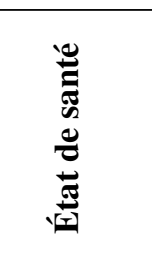 } & $\begin{array}{l}\text { Maladie chronique } \\
\text { - Pas de maladie } \\
\text { - Maladie chronique } \\
\text { - ALD }\end{array}$ & $\begin{array}{l}\text { Diagnostic } \\
\text { Diagnostic } \\
\text { Écoute }\end{array}$ & $\begin{array}{l}\text { Écoute } \\
\text { Écoute } \\
\text { Diagnostic }\end{array}$ & $\begin{array}{l}\text { Formation continue } \\
\text { Intégrité } \\
\text { Intégrité }\end{array}$ \\
\hline & $\begin{array}{l}\text { Fréquence de consultation du MT } \\
-\leq 2 \text { fois par an } \\
->3 \text { fois par an }\end{array}$ & $\begin{array}{l}\text { Diagnostic } \\
\text { Diagnostic }\end{array}$ & $\begin{array}{l}\text { Écoute } \\
\text { Écoute }\end{array}$ & $\begin{array}{l}\text { Formation continue } \\
\text { Intégrité }\end{array}$ \\
\hline \multirow{3}{*}{ 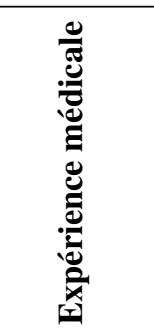 } & $\begin{array}{l}\text { Durée du suivi avec MT } \\
-\leq 4 \text { ans } \\
->5 \text { ans }\end{array}$ & $\begin{array}{l}\text { Diagnostic } \\
\text { Diagnostic }\end{array}$ & $\begin{array}{l}\text { Intégrité } \\
\text { Écoute }\end{array}$ & $\begin{array}{l}\text { Écoute } \\
\text { Intégrité }\end{array}$ \\
\hline & $\begin{array}{l}\text { Rencontre avec un bon docteur } \\
\text { - Oui } \\
\text { - Non }\end{array}$ & $\begin{array}{l}\text { Diagnostic } \\
\text { Diagnostic }\end{array}$ & $\begin{array}{c}\text { Écoute } \\
\text { Formation continue }\end{array}$ & $\begin{array}{l}\text { Intégrité } \\
\text { Écoute }\end{array}$ \\
\hline & $\begin{array}{l}\text { Rencontre avec un mauvais docteur } \\
\text { - Oui } \\
\text { - Non }\end{array}$ & $\begin{array}{l}\text { Diagnostic } \\
\text { Diagnostic }\end{array}$ & $\begin{array}{l}\text { Écoute } \\
\text { Intégrité }\end{array}$ & $\begin{array}{l}\text { Intégrité } \\
\text { Écoute }\end{array}$ \\
\hline
\end{tabular}

CSP : Catégories socio-professionnelles. Catégorie 1 : Ouvriers employés agriculteurs artisans ; Catégorie 2 : Professions supérieures et intermédiaires ; Catégorie 3 : Étudiants et inactifs. MT : médecin traitant.

Par rang de classement, l'écoute a été la qualité la plus appréciée dans cette population.

La coopération entre les soignants, la décision partagée et les conseils de prévention ont été mieux notés par les personnes exerçant une profession médicale ou paramédicale $(p \leq 0,05)$. À un moindre degré de significativité, les qualités d'écoute et la formation des étudiants ont également été mieux notées $(p<0,10)$. Par rang de classement, l'écoute a été la qualité la plus appréciée dans cette population, devant les capacités diagnostiques/thérapeutiques du médecin et son implication dans la formation médicale continue.

\section{Recours aux soins}

Les patients qui consultaient peu leur médecin ( $\leq 2$ fois par an) accordaient plus d'importance à 
l'indépendance vis-à-vis des laboratoires pharmaceutiques (moyenne $=1,40 v s .1,67 ; p=0,02$ ) que les patients consultant régulièrement leur médecin. À la limite de la significativité, les patients les plus consultants octroyaient plus d'importance au suivi des recommandations (moyenne $=2,05 v s .1,87, p=$ $0,08)$.

Les patients suivis depuis plus de cinq ans par le même médecin traitant attribuaient plus de valeur à l'écoute de la part de leur médecin que les autres patients (moyenne $=3,16 v s$. 2,98, $p=0,05$ ) et la coopération entre les soignants avait plus d'importance également pour eux (moyenne $=2,58 v s .2,41, p=$ $0,10)$.

\section{Ełat de santé du patient}

La note moyenne de la qualité d'écoute croissait progressivement entre les patients sans maladie chronique, les patients avec une maladie chronique (sans ALD) et les patients ayant une ALD (tableau III et figure 2). D'autres qualités ont été plus fréquemment observées parmi les patients ayant une maladie chronique : le suivi des recommandations, l'informatisation du cabinet médical, la décision partagée, les conseils de prévention et l'intégrité du médecin (tableau III). Après prise en compte des facteurs de confusion, les capacités d'écoute et de suivi des recommandations restaient les qualités les plus souvent citées chez les patients atteints d'une maladie chronique (figure 2). A la limite de la significativité statistique, les conseils de prévention ont été davantage cités dans cette population.

En terme de rang de classement, l'écoute était la vertu cardinale pour les patients ayant une maladie chronique et encore plus pour les patients en ALD, alors qu'elle était en deuxième position dans la population générale. Le regroupement des qualités par domaines a montré des résultats concordants avec les données ci-dessus : la moyenne des qualités relationnelles différait entre les patients, avec un gradient d'importance entre les patients sans traitement, les patients ayant un traitement chronique et les patients ayant une ALD. La notation des autres domaines de qualités ne différait pas entre les patients.

\section{Discussion}

\section{Résultats principaux}

Les trois principales qualités du bon médecin étaient, selon les patients interrogés, ses capacités à réaliser un bon diagnostic, son écoute et son intégrité. L'état de santé du patient, en particulier la présence d'une maladie chronique, était un facteur qui influait sur l'importance des qualités attendues chez un bon médecin. Un gradient de sévérité de la maladie chronique était associé à des exigences d'écoute et de qualités relationnelles accrues. D'autres facteurs sociodémographiques ou liés à l'expérience médicale du patient semblaient influer sur les qualités attribuables au bon médecin : les caractéristiques socio-démographiques, l'exercice d'une profession médicale ou paramédicale étaient plus souvent associés à une importance accrue accordée aux qualités d'écoute du médecin plutôt qu'aux capacités du médecin à réaliser un bon diagnostic. Les qualités médicales et relationnelles étaient les plus sujettes à variation, tandis que les qualités éthiques ou organisationnelles étaient peu influencées par le profil des patients.

\section{Atouts et limites}

Le premier atout de cette étude est son originalité. Cette étude est à notre connaissance la seule ayant pour objectif principal de rechercher les facteurs individuels influençant les qualités attribuées au bon médecin. Le questionnaire de l'étude a été spécifiquement développé et a inclus des facteurs connus (variables sociodémographiques) et non connus (maladie chronique, parentalité, rencontre antérieure avec un bon/mauvais médecin) pouvant influer sur les qualités attribuables au bon docteur. Les refus de participer à l'étude ont été rares (moins de $10 \%$ ), limitant les biais de non-réponses. Le recrutement, à partir d'une base de données d'appels médicaux, a permis de sélectionner un panel de population varié et comparable à la population nationale en termes d'âge (moyenne $=43,5$ ans vs. 40,1 ans en France), et d'état de santé : $24,5 \%$ des patients de l'échantillon avaient une maladie chronique vs. $20 \%$ en 


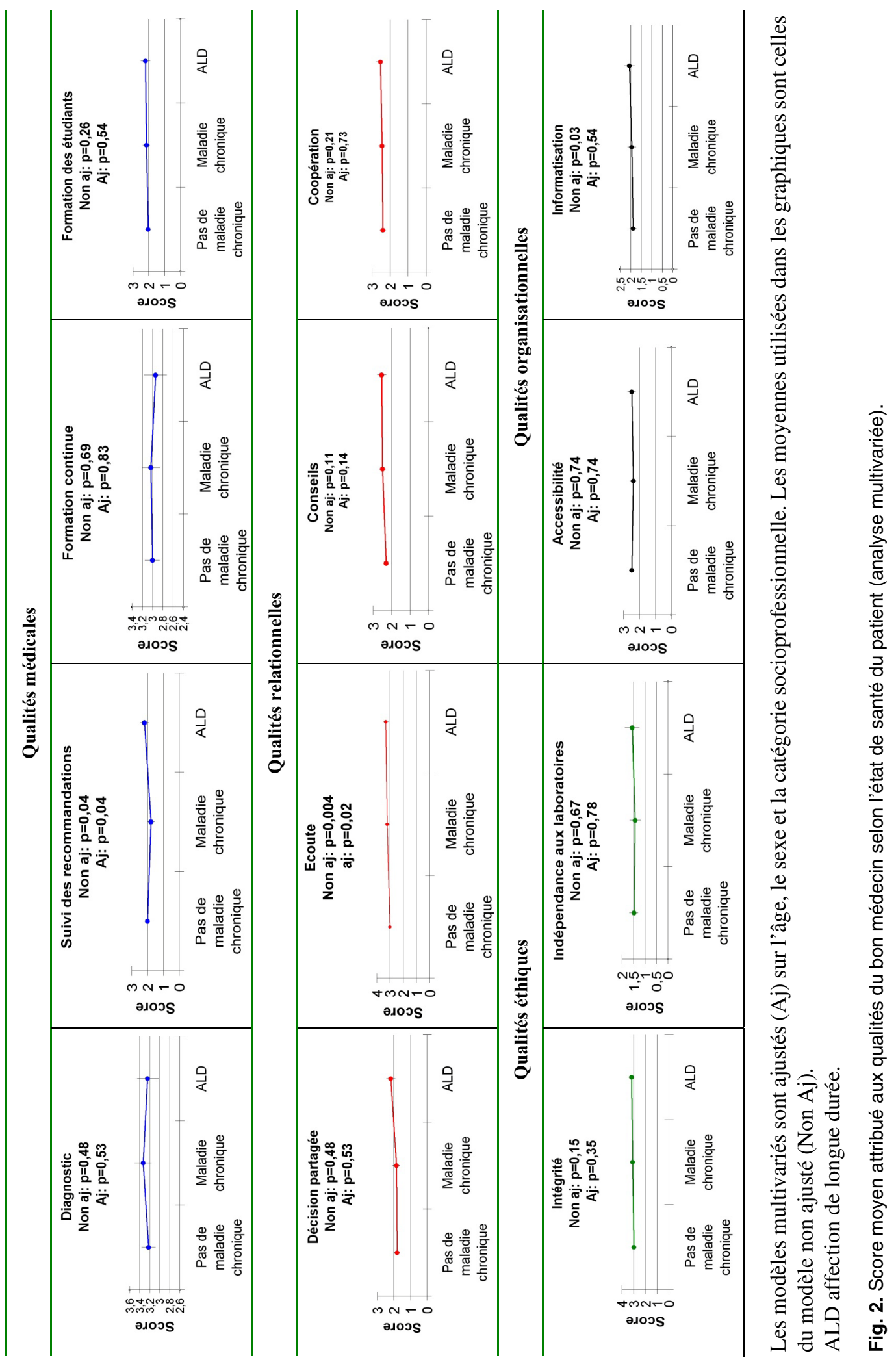


France en $2007^{[19]} ; 15,9 \%$ des patients avaient une ALD versus $14,3 \%$ en France en $2009^{[20]}$. De même, $4 \%$ des patients étaient diabétiques sous traitement vs. 3,6 \% en France métropolitaine en $2005^{[21]}$; $5,3 \%$ des patients ont déclaré avoir (eu) un cancer $v s$. $3,4 \%$ en France métropolitaine en $2002^{[22]}$. En revanche, l'utilisation d'une base de données d'appels d'urgence pouvait sélectionner des patients n'ayant pas de suivi régulier avec un médecin traitant. Cette limite a été partiellement prise en compte par les critères d'inclusion des patients ( $c f$. chapitre méthodes). En outre, notre échantillon était constitué majoritairement de femmes (70\%). Ceci pourrait être dû au fait que les femmes appellent plus souvent que leur conjoint le médecin de garde et/ou qu'elles soient plus facilement joignables durant la journée. Par ailleurs, notre échantillon «sur-représentait» les bénéficiaires de la CMUc $(11,3 \%$ vs. $6,5 \%$ en France ou 5,5\% en Haute Garonne ${ }^{[23]}$ et comprenait peu d'ouvriers/agriculteurs. Ceci pourrait s'expliquer par le recrutement important en zones franches urbaines, et la forte proportion de femmes incluses. L'utilisation de cette base de données a pu exclure les patients consultant exclusivement leur médecin traitant pour leurs problèmes de santé. Le recrutement téléphonique a limité l'inclusion aux patients qui avaient un téléphone, étaient francophones et disponibles en journée et a pu favoriser certains profils (personnes sans emploi ou invalides, par exemple). Il est également possible que les réponses aient pu être influencées par des biais de mémorisation (par exemple la variable de durée de suivi avec le médecin traitant) ou des biais d'interprétation des questions. D'autres qualités auraient pu être explorées comme l'empathie du médecin. De plus, il a été choisi de poser une seule question par qualité explorée pour limiter le temps d'administration du questionnaire. Ce point pourrait être discuté pour certaines qualités telles que « la capacité à bien diagnostiquer et bien traiter les maladies ». Pour certains auteurs, les données recueillies par les échelles de Likert sont des variables ordinales, ce qui ne permet pas le calcul des moyennes et des écart-types ${ }^{[24]}$. Pour cela, les statistiques descriptives ont également représenté la distribution des réponses par qualité (pourcentages de patients qui cotent «pas ou peu important », « très important », etc.). Les tests non paramétriques ont comparé la moyenne des rangs des réponses dans les différents groupes. Les résultats ont montré la $p$ value globale du test par qualité, ainsi que les moyennes et écart-type des réponses sous l'hypothèse d'une distribution normale des données ${ }^{[25]}$. Les qualités psychométriques du questionnaire ont été peu étudiées dans ce travail. En plus de la fidélité du questionnaire, d'autres mesures de validité auraient pu être mesurées. Dans ce travail, la valeur du coefficient alpha de Cronbach a été considérée comme acceptable. Concernant le profil des patients, certains facteurs tels que l'origine ethnique des patients n'ont pas été étudiés malgré une influence suggérée par des études outre-atlantiques. Pourtant, les patients de la même origine ethnique que leur médecin auraient plus confiance en lui ${ }^{[26]}$. Aux Etats-Unis, l'origine afro-américaine des patients semblerait orienter le comportement des médecins vers une relation plus paternaliste ${ }^{[27]}$. Dans le contexte français de notre étude, l'origine ethnique des patients nous a semblé plus délicate et moins pertinente à aborder dans le questionnaire. Les croyances religieuses, les sous-groupes des différentes maladies chroniques, le champ de la pédiatrie, les particularismes régionaux, le profil psychologique des patients, n'ont pas été étudiés. Ces variables pourraient faire l'objet d'études ultérieures. De même, ce travail explore les qualités du médecin à partir de «mots ». Il serait intéressant d'étudier ce que les patients entendent par ces mots. Les représentations semblaient parfois être différentes entre les médecins et les patients: Par exemple, «l'indépendance des médecins vis-à-vis de l'industrie pharmaceutique » ne semblait pas être perçue comme une qualité par plusieurs patients lors des échanges accompagnant l'administration orale des questionnaires: "dommage, c'est important pour les médecins de se former». Ils ne semblaient pas percevoir la dimension éthique associée à cette question, alors même que l'étude était réalisée au cours de l'affaire dite du « Médiator». Enfin, plusieurs commentaires ont été recueillis en fin de questionnaire mais n'ont pas été pris en compte dans l'analyse. Quelques exemples : "Ils ne sont pas avec nous en consultation, le téléphone sonne sans cesse »; 
«je suis plus à l'aise avec une femme même si les hommes sont souvent meilleurs»; "l'essentiel, c'est que mon médecin m'aime et qu'il aime son travail»; «les magasines médicaux dans les salles d'attente ne sont pas bons quand on est " hypocondriaque »; «si j'attends trop longtemps une consultation, ma tension monte»; «un bon médecin doit nous faire comprendre qu'on est en sursis tout en nous parlant d'autres choses ». Cela suggère l'intérêt d'une étude qualitative pour explorer l'évolution des perceptions du bon médecin au fil des expériences.

\section{Validité externe}

A notre connaissance, notre étude est la première avec cet objectif principal, même si plusieurs études ont décrit les qualités attendues d'un bon médecin dans des pays et des populations différents. Les qualités citées pouvaient différer selon les pays considérés : l'étude américaine de McBride ${ }^{[28]}$ citait comme qualité à prendre en compte le coût du traitement pour le patient. L'étude asiatique de Fones à Singapour ${ }^{[29]}$ identifiait le respect et l'engagement dans la formation médicale continue. Des études interrogeant des professeurs et des étudiants ont mis en avant l'importance de la formation continue ${ }^{[30]}$ et de la formation des étudiants ${ }^{[31]}$. Ces deux qualités ont également été mieux notées dans notre étude par les soignants et les étudiants. Les compétences cliniques et les connaissances théoriques étaient citées comme étant les qualités principales dans plusieurs études portant exclusivement sur les médecins. Les qualités d'empathie, d'écoute et de respect étaient citées plus fréquemment dans plusieurs études portant sur les patients ${ }^{[18]}$. Une étude suggérait que les hommes accordent moins d'importance à la prévention et à la morale des médecins que les femmes ${ }^{[28]}$. Dans cette même étude, de hauts revenus étaient prédictifs d'une importance accrue accordée par le patient à l'écoute, à l'éthique et à la qualité du diagnostic. Dans notre étude, le sexe et la catégorie socio-professionnelle des patients n'influençaient pas les qualités attendues de diagnostic, d'écoute et d'intégrité du médecin. La comparaison avec les travaux de la littérature est toutefois complexe car les qualités proposées, tout en étant parfois proches ne sont pas forcément les mêmes (par exemple, peut-on considérer comme équivalent la morale, l'éthique, l'intégrité, l'honnêteté ?). La traduction des termes anglophones, la spécificité de certaines populations étudiées, les modalités de questionnement et de recueil des données (annuaire téléphonique, université, service de médecine, salle de consultation, usager d'un type de clinique) rendent les comparaisons délicates. Pour autant, et de façon concordante aux résultats de notre étude, les patients semblent accorder une importance prioritaire aux qualités médicales et techniques de leur médecin ${ }^{[32]}$. Être médecin et a fortiori être un bon médecin, c'est avant tout être un "savant en médecine ${ }^{[33]}$. Nos résultats suggèrent que ces qualités évoluent dans le temps (patients plus âgés) et selon certaines spécificités de la relation médicale (accompagnement au cours d'une maladie chronique par exemple).

\section{Perspectives}

Dès 1957, Michel Balint écrivait : «le médicament le plus fréquemment utilisé en médecine générale est le médecin lui-même » ${ }^{[34]}$. Dans la perspective d'une approche centrée sur le patient, il importe que le médecin s'adapte aux besoins et aux attentes du patient. Le bon médecin devra ainsi adopter une souplesse, une adaptabilité dans son action de soigner. Cette flexibilité du «savoir être» implique trois notions. Tout d'abord, une habileté relationnelle et des compétences de communication qu'il est important d'enseigner et de valoriser chez les soignants, dès le début des études médicales. Ensuite, une connaissance singulière du patient, que le médecin acquiert au fil du temps, par un suivi régulier. Enfin, la connaissance globale de la subjectivité de patients présentant telles ou telles caractéristiques (état de santé, parcours médical, type de personnalité, etc.). Face à un patient souffrant d'une maladie chronique, et particulièrement si la maladie est sévère (bénéficiaire de l'ALD), la qualité et le temps d'écoute devraient être une préoccupation essentielle du médecin. Face à une personne âgée, la coopération entre soignants et la qualité d'écoute seraient à valoriser. Pour aller plus loin, d'autres travaux permettant 
de mieux comprendre les déterminants de la relation médecin-patient seraient à promouvoir.

Plusieurs documents ont proposé une orientation que pourrait prendre l'éducation médicale ${ }^{[35,36]}$ et le principe de responsabilité sociale en est le principe fondateur. Nos travaux espèrent apporter des arguments en faveur d'une culture de la responsabilité sociale au cours des études médicales, passant par la recherche du bien-être du patient, le développement de la pédagogie centrée sur le patient et des principes de justice sociale. Cela nécessite une offre de formation de qualité en «relation médecin-patient», appuyée par les données de la science, l'expérience, la maladie, l'analyse des conflits, des discours, etc. Cette compétence est nécessaire à une satisfaction du patient et à la prise en compte de ses besoins. La mise en application des principes essentiels de l'exercice médical laisse une place de choix aux sciences humaines et sociales, et fait largement appel au «professionnalisme médical».

\section{Contributions}

Grégory Reix, Gladys Ibanez, Anne Marie ont contribué à la conception du protocole de recherche. Grégory Reix a mené le recueil et le traitement des données. Grégory Reix, Gladys Ibanez, Julien Le Breton ont procédé à l'analyse des données. Grégory Reix, Julie Chastang, Erik Bernard, Claire Rondet ont participé à la rédaction de l'article. Anne Marie Magnier et Eric Pautas ont dirigé la recherche.

\section{Déclaration d'intérêts}

Les auteurs ne déclarent aucun conflit d'intérêt en lien avec le contenu de cet article

\section{Approbation éthique}

Une déclaration à la Commission nationale de l'informatique et des libertés (CNIL) pour autorisation d'évaluation des pratiques de soins a été déposée le 11 avril 2011. Le consentement du patient était systématiquement demandé en début d'entretien téléphonique. Les réponses ont été anonymisées à l'aide d'un identifiant numérique.

\section{Références}

1. Boelen C, Heck JE. Defining and measuring social accountability of medical schools. Genève, Suisse: Organisation mondiale de la Santé, 1995 [On-line]. Disponible sur : http://whqlibdoc.who.int/hq/ 1995/WHO_HRH_95.7.pdf

2. Meili R. La responsabilité sociale: au cœur de la médecine familiale. Can Fam Physician 2013; 59:344-5.

3. Bidaut-Russell M, Gabriel SE, Scott CG, Zinsmeister AR, Luthra HS, Yawn B. Determinants of patient satisfaction in chronic illness. Arthritis Rheum 2002;47:494-500.

4. Schönbächler G. Placebo. Forum Med Suisse 2007; 7:205-10.

5. Sablik Z, Samborska-Sablik A, Drożdż J. Universality of physicians' burnout syndrome as a result of experiencing difficulty in relationship with patients. Arch Med Sci 2013;9:398-403.

6. Grimaldi A, Cosserat J. La relation médecin-malade. Paris : Elsevier Masson, 2004.

7. Moira AS. Effective physician-patient communication and health outcomes: a review. Can Med Assoc J 1995;152:1423-33.

8. Lewin SA, Skea ZC, Entwistle V, Zwarenstein M, Dick J. Interventions for providers to promote a patient-centred approach in clinical consultations. Cochrane Database Syst Rev 2001;4:CD003267.

9. Kamrowska A. Job burn-out. Pol. Merkur. Lekarski. 2007;23:317-9.

10. Bulletin officiel $n^{\circ} 22$ du 7 juin 2007 . Ministère de l'enseignement supérieur et de la recherche, 2007. [On-line]. Disponible sur: http:// www.education.gouv.fr/bo/2007/22/ MENS0753287A.htm

11. General Medical Council I Tomorrow's Doctors online, 2009. [On-line]. Disponible sur: http:// www.gmc-uk.org/education/undergraduate/ tomorrows_doctors_2009.asp

12. Maudsley G, Williams EMI, Taylor DCM. Junior medical students' notions of a « good doctor» and related expectations: a mixed methods study. Med Educ 2007;41:476-86. 
13. Abu-Hilal M, Morgan EC, Lewis G, McPhail M, Malik HZ, Hocken D. What makes a good doctor in the 21 st century? A qualitative study. Br J Hosp Med 2006;67:375-7.

14. Fasce E, Echeverría M, Matus O, Ortiz L, Palacios S, Soto A. [Professionalism of physicians from the point of view of physicians and students]. Rev Med Chil 2009;137:746-52.

15. SOS Médecins. [On-line]. http://www.sosmedecins-france.fr/

16. Insee - Définitions et méthodes - Les nomenclatures des professions et catégories socioprofessionnelles. [On-line]. Disponible sur : http://www.insee.fr/fr/ methodes/default.asp?page=nomenclatures/ pcs.htm

17. Ibanez G, Cornet P, Minguet C. Qu'est-ce qu'un bon médecin ? Pédagogie Médicale 2010;11:151-65.

18. Sehiralti M, Akpinar A, Ersoy N. Attributes of a good physician: what are the opinions of first-year medical students? J Med Ethics 2010;36:121-5.

19. Ministère du Travail, de l'Emploi et de la Santé. Plan pour l'amélioration de la qualité de vie des personnes atteintes de maladies chroniques 2007-2011. [Online]. Disponible sur: http://www.sante.gouv.fr/ plan-pour-l-amelioration-de-la-qualite-de-viedes-personnes-atteintes-de-maladies-chroniques2007-2011.html

20. Assurance Maladie. Prévalence des Affections Longues Durées. [On-line]. Disponible sur : http:// www.ameli.fr/l-assurance-maladie/statistiques-etpublications/donnees-statistiques/affection-de-longue-duree-ald/prevalence/frequence-des-ald-au-3112-2009.php

21. Institut National de la Statistique et des Études Économiques. Les patients diabétiques traités en Guyane. [On-line]. Disponible sur : http://insee.fr/ $\mathrm{fr} /$ themes/document.asp?reg_id=25\&ref_id=13803

22. Réseau National Alimentation Cancer Recherche. Prévalence cancers. [On-line]. Disponible sur: http://www.inra.fr/nacre/pendant_le_cancer/ vous_informer_sur/prevalence_cancers

23. Institut National de la Statistique et des Études Économiques. Bénéficiaires de la couverture maladie universelle (CMU). [On-line]. Disponible sur : http://www.insee.fr/fr/themes/tableau.asp?reg_id =7\&ref_id=revtc04605

24. Jamieson S. Lickert scales: how to (ab)use them. Med Educ 2004;38:1212-8.
25. Carifio J, Perla R. Resolving the 50-year debate around using and misusing the Likert scales. Med Educ 2008;42:1150-2

26. Tarrant C, Stokes T, Baker R. Factors associated with patients' trust in their general practitioner: a crosssectional survey. Br J Gen Pract 2003;53:798-800.

27. Cooper-Patrick L, Gallo JJ, Gonzales JJ, Vu HT, Powe NR, Nelson C, et al. Race, gender, and partnership in the patient-physician relationship. JAMA 1999;282:583-9.

28. McBride CA, Shugars DA, DiMatteo MR, Lepper HS, O’Neil EH, Damush TM. The physician's role. Views of the public and the profession on seven aspects of patient care. Arch Fam Med 1994;3: 948-53.

29. Fones CS, Kua EH, Goh LG. « What makes a good doctor? » views of the medical profession and the public in setting priorities for medical education. Singapore Med J 1998;39:537-42.

30. Hur Y, Kim S. What qualities do medical school applicants need to have? secondary publication. Yonsei Med. J 2009;50:427-36.

31. Wright SM, Kern DE, Kolodner K, Howard DM, Brancati FL. Attributes of excellent attendingphysician role models. N. Engl. J. Med 1998; 339:1986-93.

32. Fung $\mathrm{CH}$, Elliott MN, Hays RD, Kahn KL, Kanouse DE, McGlynn EA, et al. Patients' preferences for technical versus interpersonal quality when selecting a primary care physician. Health Serv Res 2005; 40:957-77.

33. Geoffroy M. Un bon médecin : Pour une éthique des soins. Paris : Editions de La Table Ronde, 2007.

34. Balint M. Le Médecin, son malade et la maladie. Paris: $3^{\mathrm{e}}$ éd. Payot ; 2003.

35. Santé Canada. Social accountability: a vision for Canadian medical schools. Ottawa, ON: Santé Canada; 2001. [On-line]. Disponible sur: www.afmc.ca/pdf/ pdf_sa_vision_canadian_medical_schools_en.pdf.

36. Buchman S. Making good choices. Can Fam Physician. 2011;57:1477-8.

Correspondance et offprints : Gladys IBANEZ, Département de Médecine Générale, Faculté de Médecine, Université Pierre et Marie Curie (UPMC), Paris Sorbonne, 27 rue de Chaligny, 75012 Paris, France. Mailto : gladys.ibanez@upmc.fr 


\section{ANNEXE : Questionnaire de l'étude}

\section{Qualités du bon médecin}

\begin{tabular}{|c|c|c|c|c|}
\hline Vos critères du bon médecin : Pour vous... & $\begin{array}{c}\text { Pas/peu } \\
\text { important }\end{array}$ & $\begin{array}{l}\text { D'importance } \\
\text { moyenne }\end{array}$ & $\begin{array}{c}\text { Très } \\
\text { important }\end{array}$ & $\begin{array}{c}\text { Extrêmement } \\
\text { important }\end{array}$ \\
\hline \multicolumn{5}{|l|}{ Le bon médecin est capable de diagnostiquer une maladie et de la traiter } \\
\hline \multicolumn{5}{|c|}{$\begin{array}{l}\text { S'il existe plusieurs possibilités de traitement, le bon médecin vous laisse le choix dans la } \\
\text { façon de vous soigner }\end{array}$} \\
\hline \multicolumn{5}{|c|}{ Le bon médecin suit les recommandations officielles pour vous soigner } \\
\hline \multicolumn{5}{|l|}{ Le bon médecin prend le temps nécessaire pour vous écouter } \\
\hline \multicolumn{5}{|l|}{ Le bon médecin vous donne des conseils pour éviter les maladies } \\
\hline \multicolumn{5}{|l|}{$\begin{array}{l}\text { Le bon médecin communique bien avec les autres médecins, infirmières et autres } \\
\text { soignants pour vous soigner }\end{array}$} \\
\hline \multicolumn{5}{|l|}{ Le bon médecin continue d'apprendre, de lire, de se former } \\
\hline \multicolumn{5}{|l|}{ Le bon médecin est honnête et intègre } \\
\hline \multicolumn{5}{|l|}{ Il doit être facile et rapide d'être vu en consultation } \\
\hline \multicolumn{5}{|l|}{ Le bon médecin participe à la formation des étudiants en médecine } \\
\hline \multicolumn{5}{|l|}{ Le bon médecin est indépendant vis à vis des laboratoires pharmaceutiques } \\
\hline Le cabinet d'un bon médecin est informatisé & & & & \\
\hline
\end{tabular}

\section{Profil du répondant}

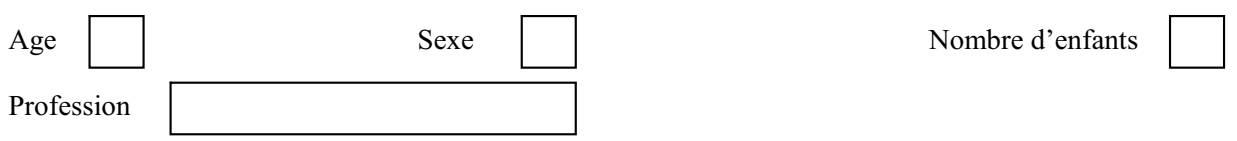

Fréquence de visite du médecin traitant
$-\quad<1$ fois/an
- [1-2] fois/an
- [3-4] fois/an
- $[4$ et $+[$ fois/an

Liste exhaustive des médicaments pris quotidiennement :

Antécédents

Maladies ou problèmes de santé actuels

CMUc

$$
\text { - Oui }
$$

- Non

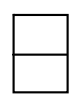

AME

- Oui

ALD

- Non

- Oui
- Non

Durée de suivi avec votre médecin traitant

Avez-vous déjà rencontré un bon médecin Avez vous déjà rencontré un mauvais médecin

- Oui

- Non

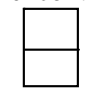

- Oui

- Non

Vous préférez que votre médecin soit

- Homme

Peu importe

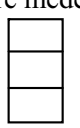

- Jeune

- Agé 